\title{
Open charm and beauty production in hadron reactions
}

\author{
G.I. Lykasova ${ }^{\mathrm{a}}$, V.V. Lyubushkin ${ }^{\mathrm{a}}$ and V.A. Bednyakov ${ }^{\mathrm{a}}$ \\ a Joint Institute for Nuclear Research, 141980, Dubna, Moscow region, Russia
}

The production of charmed and beauty hadrons in proton-proton and proton-antiproton collisions at high energies is analyzed within the modified quark-gluon string model (QGSM) including the internal motion of quarks in colliding hadrons. It is shown that using both the QGSM and NLO QCD one can describe the experimental data rather successfully in a wide region of transverse momenta. We also present some predictions for the future experiments on the beauty baryon production in $p p$ collisions at LHC energies and on the charmed meson production in $\bar{p} p$ reactions at GSI energies.

PACS: 14.65.Dw; 25.20.Lj

Keywords: Charmed quarks; Inelastic scattering: many particle final states

\section{Introduction}

Various approaches of perturbative QCD including the next-to-leading order calculations (NLO QCD) have been applied to analyze the heavy flavour particle production in hadron reactions at high energies.

Such reactions are usually analyzed within the hard parton scattering model (HPSM) suggested in [12. This model was applied to the charmed meson production both in proton-proton and meson-proton interactions at high energies, see for example [3]. The HPSM is significantly improved by applying the QCD parton approach [45, see details in [6] and references therein. Unfortunately the QCD approach including the NLO has some uncertainties related to the renormalization parameters especially at small transverse momenta $p_{t}[6]$.

In this paper we study the charmed and beauty meson production within the QGSM [7] or the dual parton model (DPM) 8 in $p p$ and $p \bar{p}$ collisions at high energies based on the $1 / N$ expansion in QCD [9]10. We show that this approach can be applied rather successfully at not very large values of $p_{t}$. In addition, we investigate the open charm and beauty baryon production in $p p$ col- lisions at LHC energies and very small $p_{t}$ within the QGSM to find new information on the sea charmed and beauty quark distributions in the proton. And at the end of the paper we use this approach to analyze the charmed meson production in the $p \bar{p}$ collision at not large energies because the obtained results would be very interesting for the future experiments at the GSI (Darmstadt) planned by the PANDA Collaboration.

\section{Charmed and beauty hadron produc- tion in $p p$ collisions at high energies}

\subsection{Heavy flavour meson production}

First, let us analyze the $D$ and $B$ meson production in the $p p$ collisions within the QGSM including the transverse motion of quarks and diquarks in colliding protons [11. As is known, the cylinder type graphs for the $p p$ collision presented in Fig.1 make the main contribution to this process [7. The left diagram of Fig.1, the so-called one-cylinder graph, corresponds to the case where two colorless strings are formed between the quark/diquark $(q / q q)$ and the diquark/quark $(q q / q)$ in colliding protons; then, after their breakup, $q \bar{q}$ pairs are created and fragmentated to a hadron, for example, $D$ meson. The right diagram of Fig.1, the so-called multicylinder graph, corresponds to creation of the same two colorless strings and many strings between sea quarks/antiquarks $q / \bar{q}$ and sea antiquarks/quarks $\bar{q} / q$ in the colliding protons. 

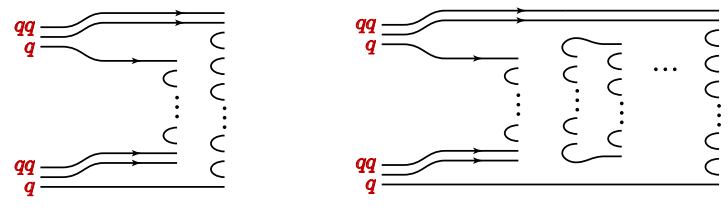

Figure 1. The one-cylinder graph (left diagram) and the multi-cylinder graph (right diagram) for the inclusive $p p \rightarrow h X$ process.

The general form for the invariant inclusive hadron spectrum within the QGSM is [12]11]

$E \frac{d \sigma}{d^{3} \mathbf{p}} \equiv \frac{2 E^{*}}{\pi \sqrt{s}} \frac{d \sigma}{d x d p_{t}^{2}}=\sum_{n=1}^{\infty} \sigma_{n}(s) \phi_{n}\left(x, p_{t}\right)$,

where $E, \mathbf{p}$ are the energy and the threemomentum of the produced hadron $h$ in the laboratory system (l.s.) of colliding protons, $E^{*}, s$ are the energy of $h$ and the square of the initial energy in the c.m.s of $p p ; x$ and $p_{t}$ are the Feynman variable and the transverse momentum of $h ; \sigma_{n}$ is the cross section for production of the $n$-Pomeron chain (or $2 n$ quark-antiquark strings) decaying into hadrons, calculated within the "eikonal approximation" [13, the function $\phi_{n}\left(x, p_{t}\right)$ has the following form [11:

$\phi_{n}\left(x, p_{t}\right)=\int_{x^{+}}^{1} d x_{1} \int_{x_{-}}^{1} d x_{2} \psi_{n}\left(x, p_{t} ; x_{1}, x_{2}\right)$,

where

$$
\begin{aligned}
& \psi_{n}\left(x, p_{t} ; x_{1}, x_{2}\right)= \\
& \quad F_{q q}^{(n)}\left(x_{+}, p_{t} ; x_{1}\right) F_{q_{v}}^{(n)}\left(x_{-}, p_{t} ; x_{2}\right) / F_{q_{v}}^{(n)}\left(0, p_{t}\right) \\
& +F_{q_{v}}^{(n)}\left(x_{+}, p_{t} ; x_{1}\right) F_{q q}^{(n)}\left(x_{-}, p_{t} ; x_{2}\right) / F_{q q}^{(n)}\left(0, p_{t}\right) \\
& +2(n-1) F_{q_{s}}^{(n)}\left(x_{+}, p_{t} ; x_{1}\right) \\
& \quad \times F_{\bar{q}_{s}}^{(n)}\left(x_{-}, p_{t} ; x_{2}\right) / F_{q_{s}}^{(n)}\left(0, p_{t}\right)
\end{aligned}
$$

and $x_{ \pm}=\left(\sqrt{x^{2}+x_{t}^{2}} \pm x\right) / 2$, $x_{t}=2 \sqrt{\left(m_{h}^{2}+p_{t}^{2}\right) / s}$,

$$
\begin{aligned}
& F_{\tau}^{(n)}\left(x_{ \pm}, p_{t} ; x_{1,2}\right) \\
& \quad=\int d^{2} k_{t} \tilde{f}_{\tau}^{(n)}\left(x_{1,2}, k_{t}\right) \tilde{G}_{\tau \rightarrow h}\left(\frac{x_{ \pm}}{x_{1,2}}, k_{t} ; p_{t}\right), \\
& F_{\tau}^{(n)}\left(0, p_{t}\right) \\
& \quad=\int_{0}^{1} d x^{\prime} d^{2} k_{t} \tilde{f}_{\tau}^{(n)}\left(x^{\prime}, k_{t}\right) \tilde{G}_{\tau \rightarrow h}\left(0, p_{t}\right) \\
& =\tilde{G}_{\tau \rightarrow h}\left(0, p_{t}\right) .
\end{aligned}
$$

Here $\tau$ means the flavor of the valence (or sea) quark or diquark, $\tilde{f}_{\tau}^{(n)}\left(x^{\prime}, k_{t}\right)$ is the quark distribution function depending on the longitudinal momentum fraction $x^{\prime}$ and the transverse momentum $k_{t}$ in the $n$-Pomeron chain; $\quad \tilde{G}_{\tau \rightarrow h}\left(z, k_{t} ; p_{t}\right)=z \tilde{D}_{\tau \rightarrow h}\left(z, k_{t} ; p_{t}\right)$, $\tilde{D}_{\tau \rightarrow h}\left(z, k_{t} ; p_{t}\right)$ is the fragmentation function $(\mathrm{FF})$ of a quark (antiquark) or diquark of flavour $\tau$ into a hadron $h$ ( $D$ meson in our case). We present the quark distributions and the $\mathrm{FF}$ in the factorized forms $\tilde{f}_{\tau}\left(x, k_{t}\right)=f_{\tau}(x) g_{\tau}\left(k_{t}\right)$, and, according to [15], $\tilde{G}_{\tau \rightarrow h}\left(z, k_{t} ; p_{t}\right)=$ $G_{\tau \rightarrow h}(z) \tilde{g}_{\tau \rightarrow h}\left(\tilde{k}_{t}\right)$, where $\tilde{\mathbf{k}}_{t}=\mathbf{p}_{t}-z \mathbf{k}_{t}$. We take the quark distributions $f_{\tau}(x)$ and the $\mathrm{FF}$ $G_{\tau \rightarrow h}(z)$ obtained within the QGSM from [12, 1617], whereas their $k_{t}$ distributions are chosen in the form suggested in 1415. (see the details in 6])

$$
\begin{aligned}
& g_{\tau}\left(k_{t}\right)=\left(B_{q}^{2} / 2 \pi\right) \exp \left(-B_{q} A\left(k_{t}\right)\right), \\
& \tilde{g}_{\tau \rightarrow h}\left(\tilde{k}_{t}\right)=\left(B_{c}^{2} / 2 \pi\right) \exp \left(-B_{c} A\left(k_{t}\right)\right),
\end{aligned}
$$

where $\left.A\left(k_{t}\right)=\left(\sqrt{k_{t}^{2}+m_{D}^{2}}-m_{D}\right)\right)$ and $m_{D t}^{2}=$ $p_{t}^{2}+m_{D}^{2}$ After the integration of eq. (4) over $d^{2} k_{t}$ we have, according to [15],

$$
\begin{aligned}
& F_{\tau}^{(n)}\left(x_{ \pm}, p_{t} ; x_{1,2}\right) \\
& \quad=\tilde{f}_{\tau}^{(n)}\left(x_{1,2}\right) G_{\tau \rightarrow h}(z) I_{n}\left(z, p_{t}\right),
\end{aligned}
$$

where $z=x_{ \pm} / x_{1,2}, I_{n}\left(z, p_{t}\right)=B_{z}^{2} /(2 \pi(1+$ $\left.\left.B_{z} m_{D}\right)\right) \exp \left(-B_{z}\left(m_{D t}-m_{D}\right)\right), B_{z}=B_{c} /(1+$ $\left.n \rho z^{2}\right), \rho=B_{c} / B_{q}$. The function $B_{z}$ also can be presented in the equivalent form $B_{z}=B_{q} /(\tilde{\rho}+$ $\left.n z^{2}\right)$, where $\tilde{\rho}=B_{q} / B_{c}$. The differential cross section $d \sigma / d p_{t}^{2}$ for $D$ mesons produced in $p p$ col- 
lisions is written in the form 12

$$
\frac{d \sigma}{d p_{t}^{2}}=\frac{\pi}{2} \sqrt{s} \sum_{n=1}^{\infty} \sigma_{n}(s) \int \phi_{n}\left(x, p_{t}\right) \frac{d x}{E^{*}} .
$$

To describe the experimental data on the $p_{t^{-}}$ spectra in the $p_{t}$ region where the NLO QCD calculation has a big uncertainty we chose $\tilde{\rho}=7$ both for the Tevatron and the LHC energies. When $B_{c}<1(\mathrm{GeV} / \mathrm{c})^{-1}$, Eq.(7) can be approximately presented in the form $\tilde{g}_{\tau \rightarrow h}\left(\tilde{k}_{t}\right) \simeq$ $a^{2} /\left(a^{2}+\tilde{k}_{t}^{2}\right)$ at $\tilde{k}_{t}^{2}<2 m_{D}$, where $a=\sqrt{2 m_{D} / B_{c}}$ [19]. This form is similar to the form for the FF of heavy quarks obtained within the perturbative QCD. Note that the function $I_{n}\left(z, p_{t}\right)$ in Eq. (8) was obtained in [15]1] on the assumption of the consequent sharing of the transverse momentum $p_{t}$ in the proton (antiproton) between $n$-Pomeron chains. It allowed us to describe rather satisfactorily the experimental data on the inclusive $p_{t}$ spectra of charmed and beauty mesons produced in $p \bar{p}$ collisions at moderate values of the transverse momentum $p_{t}<10 \mathrm{GeV} / \mathrm{c}$ 6. As is shown in [15], this version of the QGSM describes rather satisfactorily the experimental data on inclusive spectra of $D$ mesons produced in $p p$ collisions at $\sqrt{s}=27.4 \mathrm{GeV}$.

It allows us to make the predictions for inclusive $p_{t}$ spectra of $D^{0}$ and $B^{+}$mesons produced in the $p p$ collision at LHC energies and compare our results with the NLO QCD calculation for the produced charmed quarks [18, see Figs.2, 3. The solid lines in Figs.2, 3 correspond to our calculations within the QGSM, whereas the hatched regions show the calculations within the NLO QCD including uncertainties [18. A big difference between the QGSM and NLO QCD calculations at $p_{t}>10 \mathrm{GeV} / \mathrm{c}$ for $D$ and $B$ mesons can be due to the following. First, the NLO QCD calculation [18] does not include the hadronization of quarks to heavy mesons, whereas the QGSM calculation includes it. Second, we do not include the contribution of gluons and their hard scatterings off quarks and gluons which can be sizable at large values of $p_{t}$.

\subsection{Heavy flavor baryon production}

Now let us analyze the charmed and beauty baryon production in the $p p$ collision at LHC en-

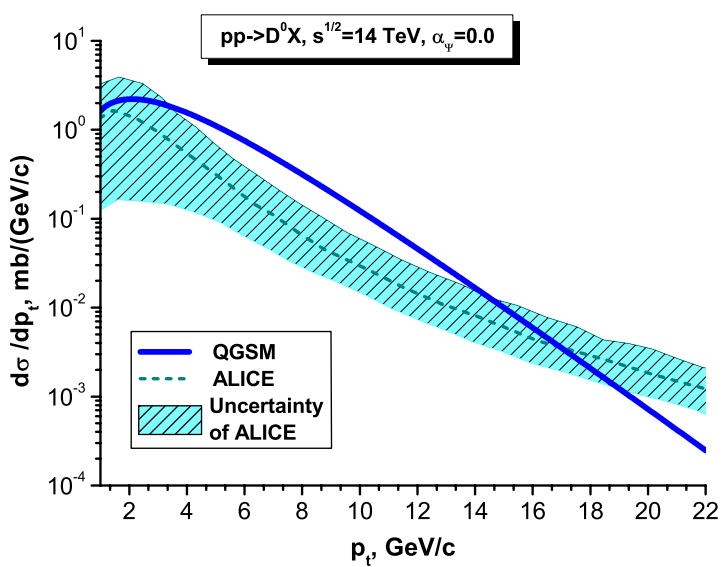

Figure 2. The inclusive spectrum for $D^{0}$ mesons produced in the $p p$ collision at the LHC energy $\sqrt{s}=14 \mathrm{TeV}$ obtained within the QGSM for charmed mesons [6] and the NLO QCD for $c$ quarks [18.

ergies and very small $p_{t}$ within the soft QCD, e.g., the QGSM. This study can be interesting for it may allow predictions for future LHC experiments like TOTEM and ATLAS and an opportunity to find new information on the distribution of sea charmed $(c)$ and beauty $(b)$ quarks at very low $Q^{2}$. According to the QGSM, the distribution of $c(\bar{c})$ quarks in the $n$th Pomeron chain (Fig.1(right)) is, see for example [1] and references therein,

$$
\begin{aligned}
& f_{c(\bar{c})}^{(n)}(x)=C_{c(\bar{c})}^{(n)} \delta_{c(\bar{c})} x^{-\alpha_{\psi}(0)} \\
& \quad \times(1-x)^{\alpha_{\rho}(0)-2 \alpha_{B}(0)+\left(\alpha_{\rho}(0)-\alpha_{\psi}(0)\right)+n-1},
\end{aligned}
$$

where $\delta_{c(\bar{c})}$ is the weight of charmed pairs in the quark sea, $C_{c(\bar{c})}^{(n)}$ is the normalization coefficient [12, $\alpha_{\psi}(0)$ is the intercept of the $\psi$ - Regge trajectory. Its value can be -2.18 assuming that this trajectory $\alpha_{\Psi}(t)$ is linear and the intercept and the slope $\alpha_{\Psi}^{\prime}(0)$ can be determined by drawing the trajectory through the $\Psi$-meson mass $m_{\Psi}=3.1$ $\mathrm{GeV}$ and the $\chi$-meson mass $m_{\chi}=3.554 \mathrm{GeV}[20$. Assuming that the $\psi$-Regge trajectory is nonlinear one can get $\alpha_{\psi}(0) \simeq 0$, which follows from 


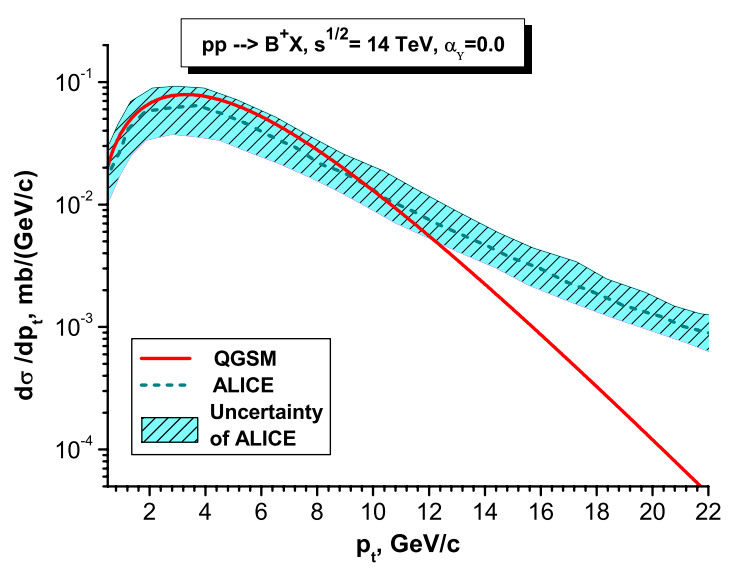

Figure 3. The inclusive spectrum for $B^{+}$mesons produced in the $p p$ collision at the LHC energy $\sqrt{s}=14 \mathrm{TeV}$ obtained within the QGSM for beauty mesons [6] and the NLO QCD for single $b$ quarks [18.

perturbative QCD, as it was shown in [21]. The distribution of $b(\bar{b})$ quarks in the $n$th Pomeron chain (Fig.1(right)) has the similar form

$$
\begin{aligned}
& f_{b(\bar{b})}^{(n)}(x)=C_{b(\bar{b})}^{(n)} \delta_{b(\bar{b})} x^{-\alpha_{\Upsilon}(0)} \\
& \quad \times(1-x)^{\alpha_{\rho}(0)-2 \alpha_{B}(0)+\left(\alpha_{\rho}(0)-\alpha_{\Upsilon}(0)\right)+n-1},
\end{aligned}
$$

where $\alpha_{\rho}(0)=1 / 2$ is the well known intercept of the $\rho$-trajectory; $\alpha_{B}(0) \simeq-0.5$ is the intercept of the baryon trajectory, $\left.\alpha_{\Upsilon}(0)\right)$ is the intercept of the $\Upsilon$ - Regge trajectory, its value also has an uncertainty. Assuming its linearity one can get $\alpha_{\Upsilon}(0)=-8,-16$, while for nonlinear $(b \bar{b})$ Regge trajectory $\alpha_{\Upsilon}(0) \simeq 0$, see details in [17. Inserting these values to the form for $f_{c(\bar{c})}^{n)}(x)$ and $f_{b(\bar{b})}^{n)}(x)$ we get the large sensitivity for the $c$ and $b$ sea quark distributions in the $n$th Pomeron chain. Note that the FFs also depend on the parameters of these Regge trajectories. Therefore, the knowledge of the intercepts and slopes of the heavymeson Regge trajectories is very important for the theoretical analysis of open charm and beauty production in hadron processes.

The information on the charmonium $(c \bar{c})$ and

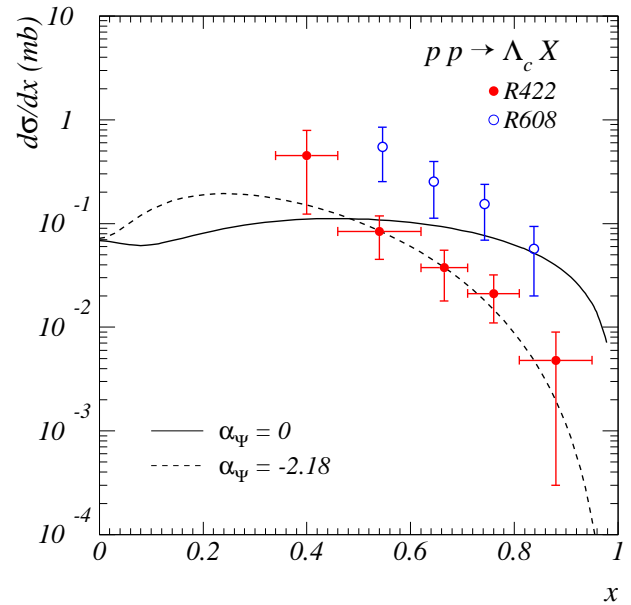

Figure 4. The differential cross section $d \sigma / d x$ for the inclusive process $p p \rightarrow \Lambda_{c} X$ at $\sqrt{s}=62 \mathrm{GeV}$.

botomonium $(b \bar{b})$ Regge trajectories can be found from the experimental data on the charmed and beauty baryon production in $p p$ collisions at high energies. For example, Fig.4 illustrates the sensitivity of the inclusive spectrum $d \sigma / d x$ of the produced charmed baryons $\Lambda_{c}$ to different values for $\alpha_{\psi}(0)$. The solid line corresponds to $\alpha_{\psi}(0)=0$, whereas the dashed curve corresponds to $\alpha_{\psi}(0)=-2.18$. Unfortunately the experimental data presented in Fig.4 have big uncertainties; therefore, one can't extract the information on the $\alpha_{\psi}(0)$ values from the existing experimental data.

A high sensitivity of the inclusive spectrum $d \sigma / d x$ of the produced beauty baryons $\Lambda_{b}$ to different values for $\alpha_{\Upsilon}(0)$ is presented in Fig.5 (left). The $p_{t}$-inclusive spectrum of $\Lambda_{b}$ has much lower sensitivity to this quantity, according to the results presented in Fig.5 (right). Actually, our results presented in Fig.5 could be considered as some predictions for future experiments at LHC. 

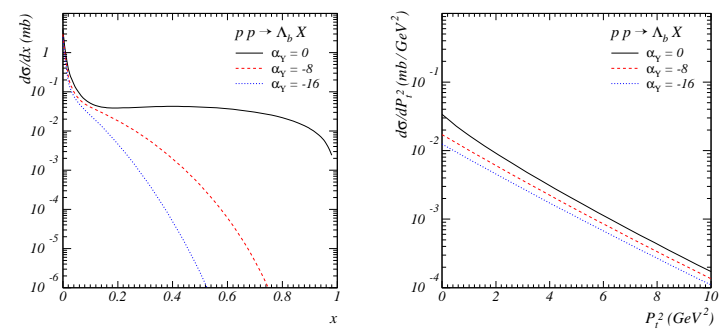

Figure 5. The differential cross section $d \sigma / d x$ (left) and $d \sigma / d P_{t}^{2}$ (right) for the inclusive process $p p \rightarrow \Lambda_{b} X$ at $\sqrt{s}=4 \mathrm{TeV}$.

$$
\begin{aligned}
E \frac{d \sigma^{p \bar{p}}}{d^{3} \mathbf{p}}= & \sigma_{1}(s)\left[(1-\omega) \phi_{1}^{p \bar{p}}\left(x, p_{t}\right)+\omega \tilde{\phi}\left(x, p_{t}\right)\right] \\
& +\sum_{n=2}^{\infty} \sigma_{n}(s) \phi_{n}^{p \bar{p}}\left(x, p_{t}\right)
\end{aligned}
$$
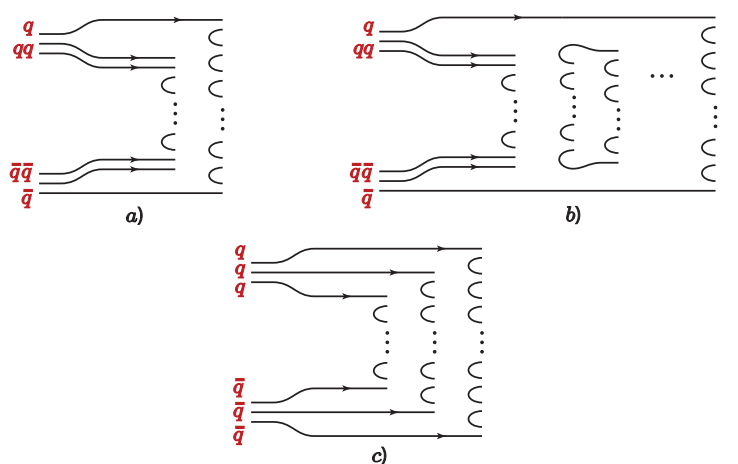

Figure 6. The one-cylinder graph (a) and the multi-cylinder graph (b), and the three-chains graph (c) for the $p \bar{p} \rightarrow h X$ inclusive process.

where $1-\omega$ is the probability of contribution of the cut one-cylinder (one-Pomeron exchange) and cut multicylinder (multiPomeron exchanges) graphs (the left and right diagrams in Fig.6), whereas $\omega$ is the probability of the contribution of the three-chain diagram to the inclusive spectrum. The value of $\omega$ can be estimated as the ratio of the $p \bar{p}$ annihilation cross section $\sigma_{p \bar{p}}^{a n n}$ to the total $p \bar{p}$ cross section $\sigma_{p \bar{p}}^{t o t}$. The cross section $\sigma_{p \bar{p}}^{t o t}$ is well known in the wide range of the initial energies to the Tevatron energy, whereas experimental data on $\sigma_{p \bar{p}}^{a n n}$ are available only for the antiproton initial energy up to $10 \mathrm{GeV}$, see [24] and references therein. However, some theoretical predictions, for example [2526], show that asymptotically $\sigma_{p \bar{p}}^{a n n}$ goes to about $2-4 \mathrm{mb}$. It corresponds to $\omega \simeq \sigma_{p \bar{p}}^{a n n} / \sigma_{p \bar{p}}^{t o t}<0.1$ at the Tevatron energy. Note that in addition to the graph of Fig.6c there can be diagrams consisting of these calculated within the QGSM has the following form: 
three chains and multicilynder chains between sea quarks and antiquarks. However, as our estimations show, their contribution to the inclusive spectrum is much smaller than the contribution from the three-chain graph (Fig.6c). Therefore, we neglect it.

The form for the function $\phi_{n}^{p \bar{p}}\left(x, p_{t}\right)$ is similar to $\phi_{n}\left(x, p_{t}\right)$ entering into (3) by replacing $F_{q_{v}}^{(n)}\left(x_{-}, p_{t} ; x_{2}\right), \quad F_{q_{v}}^{(n)}\left(0, p_{t}\right)$ to $F_{\overline{q q}}^{(n)}\left(x_{-}, p_{t} ; x_{2}\right), \quad F_{\bar{q} q}^{(n)}\left(0, p_{t}\right)$ respectively, and replacing $F_{q q}^{(n)}\left(x_{-}, p_{t} ; x_{2}\right), \quad F_{q q}^{(n)}\left(0, p_{t}\right)$ to $F_{\bar{q}}^{(n)}\left(x_{-}, p_{t} ; x_{2}\right)$ and $F_{\bar{q}}^{(n)}\left(0, p_{t}\right)$ respectively. The additional term $\tilde{\phi}\left(x, p_{t}\right)$ in (12) has the following form

$\tilde{\phi}\left(x, p_{t}\right)=3 \tilde{F}_{q_{v}}\left(x_{+}, p_{t}\right) \tilde{F}_{\bar{q}_{v}}\left(x_{-}, p_{t}\right) / \tilde{F}_{q}\left(0, p_{t}\right)$,

where $\tilde{F}_{q_{v}\left(\bar{q}_{v}\right)}\left(x_{ \pm}, p_{t}\right)=F_{q_{v}\left(\bar{q}_{v}\right)}^{(n=1)}\left(x_{ \pm}, p_{t}\right)$ and $\tilde{F}_{q}\left(0, p_{t}\right)=F_{q}^{(n=1)}\left(0, p_{t}\right)$.

The inclusive $p_{t}$ spectra of $D^{0}$ and $B^{+}$mesons produced in the $p \bar{p}$ collision at the Tevatron energy $\sqrt{s}=1.96 \mathrm{TeV}$ are presented in Figs. $(7,8)$, see 6]. The hatched regions in Figs.(7.8) show the calculations within the NLO QCD including uncertainties 29. Note that the our calculation showed that the contribution of the three-chain graph (Fg.6c) is very small at the Tevatron energy. It is due to small values of the $p \bar{p}$ annihilation cross section at very high energies [25|26].

\subsection{Charmed meson production at inter- mediate energies}

Let us now use the QGSM to analyze the inclusive charmed meson production in $\bar{p} p$ collisions at not large energies, less than $15-20 \mathrm{GeV}$ because it would be interesting for future experiments at the GSI (Darmstadt) planned by the PANDA Collaboration. At these energies the contribution of the three-chain graph (Fig.6c) can be sizable because the cross section of the $\bar{p} p$ annihilation is not small 24]. Note that at energies close to the threshold of the $D$-meson production the binary process $\bar{p} p \rightarrow \bar{D} D$ is dominant, according to 30 . Therefore, the total cross section of $D$ mesons produced in $\bar{p} p$ collisions at energies starting from the threshold is the sum of cross sections for the

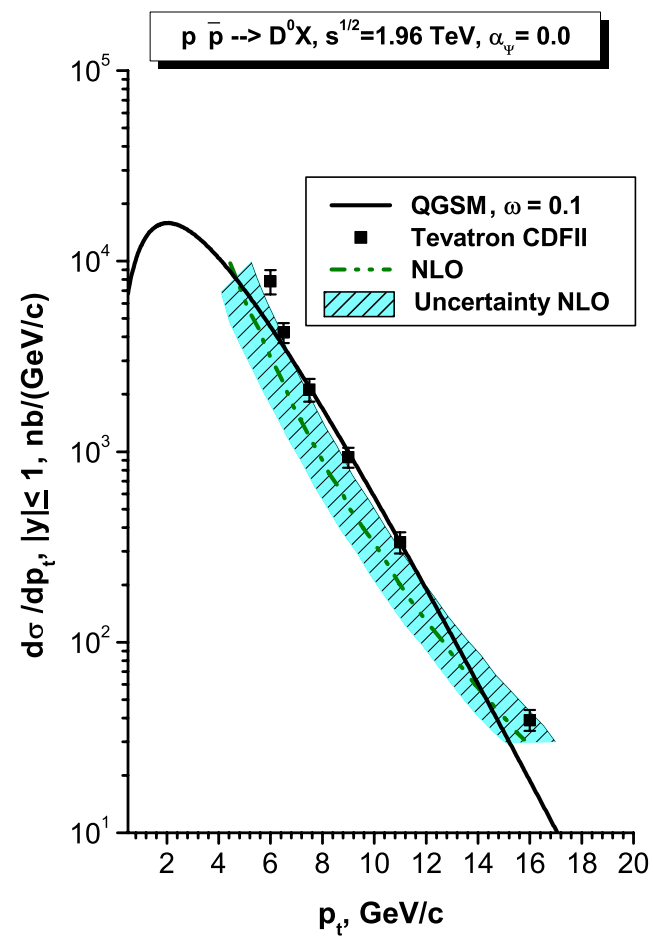

Figure 7. The inclusive $p_{t}$-spectrum for $D^{0}$ mesons produced in the $p \bar{p}$ collision at the Tevatron energy $\sqrt{s}=1.96 \mathrm{TeV}$ obtained within the QGSM [6] (the solid line) and within the NLO QCD [29] (the hatched regions). The experimental data are taken from $27 / 28]$

binary process $\sigma_{\bar{p} p \rightarrow \bar{D} D}$ and the inclusive reaction $\sigma_{\bar{p} p \rightarrow D X}$. The cross section $\sigma_{\bar{p} p \rightarrow \bar{D} D}$ was calculated in [30] within the simple Regge pole model. In Fig. 9 the cross sections $\sigma_{\bar{p} p \rightarrow \bar{D} D}$ (dashed curve) and $\sigma_{\bar{p} p \rightarrow D X}$ (dash-dotted line and dotted line) are presented as functions of the incident momentum of the antiproton in the l.s. of $\bar{p} p$. The dash-dotted curve corresponds to our calculation of the inclusive process $\bar{p} p \rightarrow D(\bar{D}) X$ with neglect of the graph of Fig.6c $(\omega=0)$, and the dotted line corresponds to the similar calculation but with the Fig.6c graph included; the probability for the three-chain graph (Fig.6c) $\omega=\sigma_{\bar{p} p}^{a n n} / \sigma_{\bar{p} p}^{t o t}$ was taken from the fit of the experimental data 


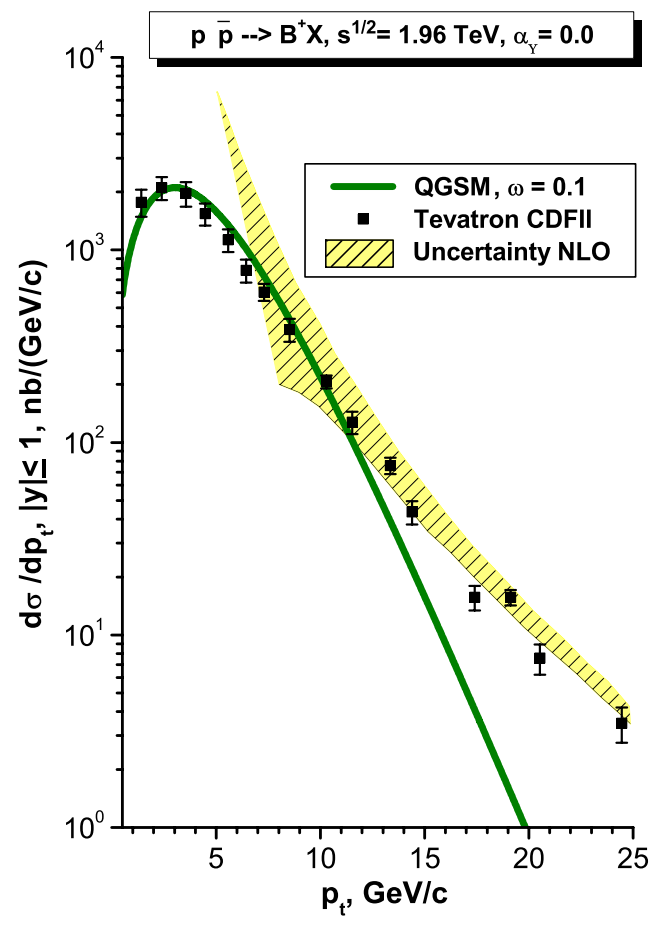

Figure 8. The inclusive $p_{t}$-spectrum for $B^{+}$ mesons produced in the $p \bar{p}$ collision at the Tevatron energy $\sqrt{s}=1.96 \mathrm{TeV}$ obtained within the QGSM [6] (the solid line) and within the NLO QCD 29] (the hatched regions). The experimental data are taken from $27 / 28$

24. The solid line is the total yield of $D$ mesons produced in $\bar{p} p$ collision.

\section{Conclusion}

We have shown that the modified QGSM including the intrinsic longitudinal and transverse motion of quarks (antiquarks) and diquarks in colliding protons allowed us to describe rather satisfactorily the existing experimental data on inclusive spectra of heavy flavour mesons produced in $p p$ collisions and to make some predictions for similar spectra at LHC energies.

To verify whether these predictions can be re-

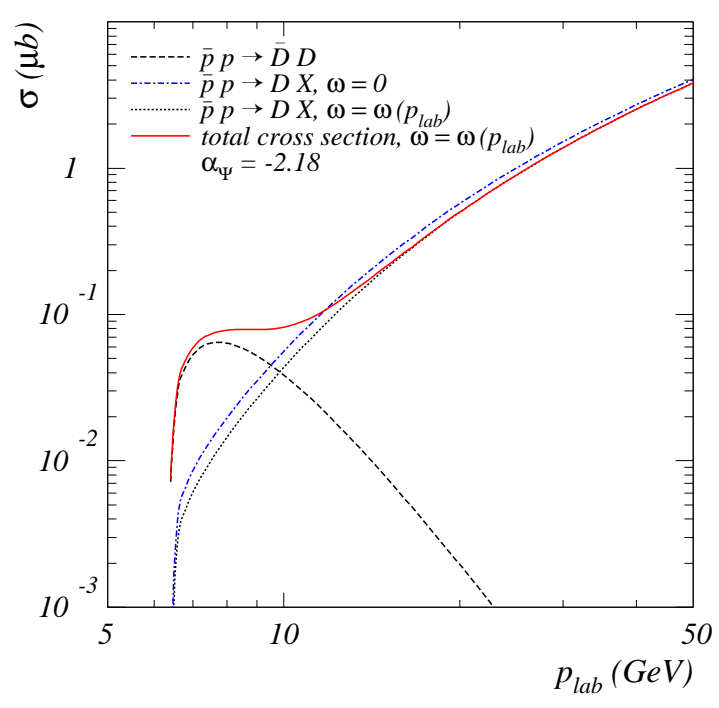

Figure 9. The cross section for $D(\bar{D})$ meson production in $\bar{p}-p$ collision as a function of the antiproton momentum $p_{l a b}$ in the l.s.

liable or not we apply the QGSM to the analysis of charmed and beauty meson production in proton-antiproton collisions at Tevatron energies including graphs like those in Fig.6c corresponding to annihilation of quarks and antiquarks in colliding $p$ and $\bar{p}$, and production of heavy flavour mesons. We got a satisfactory QGSM description $\left(p_{t}<10 \mathrm{GeV} / \mathrm{c}\right)$ of the experimental data on $p_{t}$ spectra of $D^{0}$ and $B^{+}$mesons produced in the $p \bar{p}$ collisions which were obtained by the CDFII Collaboration at the Tevatron 29 .

To describe these spectra and make some predictions for the future LHC experiments in a wide region of transverse momenta one can combine the "soft QCD" (the QGSM) at small values of $p_{t}$ with the NLO QCD at large $p_{t}$.

At the Tevatron energies the contribution of the three-chain graph (Fig.6c) to the inclusive spectra of heavy mesons is too small, as was shown in [6]; therefore, it can be neglected. However, at the antiproton energies about a few $\mathrm{GeV}$ the three-chain graph contribution is sizable and 
can amount to 30-40 percent, as is shown in Fig.9. It has also been shown that at the incident momenta of antiprotons $p_{l a b}$ above $8-9 \mathrm{GeV} / \mathrm{c}$ the inclusive production of $D$ mesons in $\bar{p} p$ collisions should be included in addition to the binary $\bar{p} p \rightarrow \bar{D} D$ process to get the total yield of $D$ mesons at $p_{l a b} \leq 14-15 \mathrm{GeV} / \mathrm{c}$. These results would be interesting for future experiments at the GSI (Darmstadt) with the antiproton beam.

We also made some predictions for future LHC forward experiments on the beauty baryon production in $p p$ collisions which can give us new information on the beauty quark distribution in the proton and very interesting information on the Regge trajectories of $(b \bar{b})$ mesons.

\section{Acknowledgments}

We thank P. Braun-Munzinger, W. Cassing, M. Deile, S. Dubnicka, A. Z. Dubnickova, A. V. Efremov, K. Eggert, D. Elia, A.Galoyan, S. B. Gerasimov, A. B. Kaidalov, Z. M. Karpova, B. Z. Kopeliovich, A. D. Martin, K. Peters, T. V. Lyubushkina, M. Poghosyan, K. Safarik, V. V. Uzhinsky and U. Wiedner for very useful discussions. This work was supported in part by the RFBR grant N 08-02-01003.

\section{REFERENCES}

1. A. V. Efremov, Yad. Fiz. 19 (1974) 179.

2. R. D. Field and R. P. Feyman, Phys. Rev. D15 (1977) 2590; R. D. Field, R. P. Feyman and G. C. Fox, Nucl. Phys. B128 (1977) 1.

3. V. A. Bednyakov, Mod.Phys.Lett. A10 (1995) 61.

4. P. Nasson, S. Dawson and R. K. Ellis, Nucl. Phys. B303 (1988) 607; ibid. B327 (1989) 49;ibid. B335 (1989) 260E.

5. B. A. Kniehl and G.Kramer, Phys.Rev. D60 (1999) 014006.

6. G. I. Lykasov, Z. M. Karpova, M. N. Sergeenko and V. A. Bednyakov, Europhys.Lett.86 (2009), 61001; arXiv:0812.3220 [hep-ph].

7. A. B. Kaidalov, Phys. Lett. B116 (1982) 459; A. B. Kaidalov and K. A. Ter-Martirosyan, Phys. Lett. B117 (1982) 247.
8. A. Capella, U. Sukhatme, C. I. Tan, J. Tran Than Van, Phys. Rep. 236 (1994) 225.

9. G. t'Hooft, Nucl.Phys. B72 (1974) 461.

10. G. Veneziano, Phys. Lett. B52 (1974) 220.

11. G. I. Lykasov, G. H. Arakelian and M. N. Sergeenko, Phys. Part. Nucl. 30 (1999) 343; G. I. Lykasov, M. N. Sergeenko, Z. Phys. C70 (1996) 455.

12. A. B. Kaidalov and O. I. Piskunova, Z. Phys. C30 (1986) 145.

13. K. A. Ter-Martirosyan, Phys. Lett. B44 (1973) 377.

14. A. I. Veselov, O. I. Piskunova, K. A. TerMartirosyan, Moscow, ITEP-176 preprint, 1990.

15. G. I. Lykasov, M. N. Sergeenko, Z. Phys. C56 (1992) 697.

16. Yu. M. Shabelsky, Yad. Fiz. 56 (1992) 2512.

17. O. I. Piskunova, Yad. Fiz. 56 (1993) 176 (Phys.Atom.Nucl. 56 (1993) 1094); ibid. 64 (2001) 392.

18. B. Alessandro, et al., (ALICE Colalboration) J. Phys. G: Nucl. Part. Phys. 32 (2006) 1295.

19. M. Cacciari and M. Greco, Phys. Rev. Lett. 73 (1994) 1586.

20. K. G. Boreskov, A. B. Kaidalov, Sov.J.Nucl.Phys. 37 (1983) 100.

21. A. B. Kaidalov, O. I. Piskunova, Sov.J.Nucl.Phys. 43 (1986) 994.

22. P.Chauvat, et al., (R608 Collaboration) Phys.Lett. B199 (1987) 304.

23. G.Bari, et al., (R422 Collaboration) Nuovo Cim. A104 (1991) 571.

24. V. V. Uzhinsky and A. S. Galoyan hep-ph/0212369 (2002).

25. E. Gostman and S. Nussinov, Phys. Rev. D22 (1980) 624.

26. B. Z. Kopeliovich and B. G. Zakharov, Phys. Lett.B211 (1986) 221.

27. D. Acosta, et al., (CDF Collaboration) Phys. Rev. D71 (2005) 032001.

28. A. Abulencia, et al., (CDF Collaboration) Phys.Rev. D75 (2007) 012010.

29. D. Acosta, et al., (CDF Collaboration) Phys. Rev. Lett. 91 (2003) 241804.

30. A. B. Kaidalov, P. E. Volkovitsky, Z.Phys. C63 (1994) 517. 